\title{
Biological Entities Isolated from Two Stratosphere Launches-Continued Evidence for a Space Origin
}

\author{
Wainwright $\mathrm{M}^{1,3 *}$, Rose $\mathrm{CE}^{2}$, Baker $\mathrm{AJ}^{2}$, Wickramasinghe $\mathrm{NC}^{3}$ and Omairi $\mathrm{T}^{1}$
}

${ }^{1}$ Department of Molecular Biology and Biotechnology, University of Sheffield, Sheffield S10 2TN, UK

${ }^{2}$ Leonardo Centre for Tribology, University of Sheffield, Sheffield S10 2TN, UK

${ }^{3}$ Buckingham Centre for Astrobiology, University of Buckingham, Buckingham MK18 1EG, UK

\begin{abstract}
Here, we provide images of a variety of biological entities which we have sampled from the stratosphere at heights of between $23-27 \mathrm{Km}$. The biological entities have unusual morphology and are generally not representatives of known terrestrial organisms. Analysis using EDX shows that all of the BEs contain only $C$ and $O$ and are not associated on the sampling stubs with pollen, grass or other terrestrial organisms. Images are provided of some of the biological entities which were sampled from our first and third successful sampling trips, a second trip isolated inorganic micrometeorites, but not biological entities. We conclude that the evidence points to a space origin for these stratosphere-derived biological entities.
\end{abstract}

Keywords: Biological entities; EDX; Space; Control flight; Stratosphere

\section{Introduction}

We have previously reported Scanning Electron Images (SEM) of Biological Entities (BEs) isolated from the stratosphere using a balloonlaunched sampler [1,2]. These reports provide evidence for a limited number, but wide variety of BEs which EDX analysis shows are not inorganic particles made up of elements such as silicon, calcium and iron, but are instead comprised of only carbon and oxygen, i.e. they have an organic composition. We have previously suggested that these particles are incoming to Earth from space and therefore do not have a contemporary terrestrial origin; the possibility exist however, that they had a prehistoric terrestrial origin and are now returning to Earth. Our claim favoring a space origin for these particles is based on the following:

1) The BEs occur in very low numbers as isolated particles on the carbon sampling stubs which were exposed to the stratosphere. With the exception of a single diatom frustule fragment, known terrestrial organisms commonly found on Earth (e.g. grass seeds and pollen) are however, not sampled from this source. In addition material having the typical morphology and EDX signature of volcanic dust [3] has never been seen on the sampling stubs.

2) The observed stratosphere-derived BEs is unusual and (with the exception of the diatom fragment) cannot be identified, by us, as terrestrial organisms (e.g. marine or terrestrial alga or protozoa).

3) The biological entities are often associated with impact craters caused by inorganic, space-derived cosmic dust.

4) Some of the BEs themselves also produced impact craters on the carbon sampling stubs, again suggesting that they are incoming to Earth from space.

5) The BEs, and/or the inorganic masses on which they are found, exceed 5 microns, the size limit above which it is generally assumed that particles cannot be transported from the troposphere to the stratosphere [4]. It is also worth noting that stratospheric dust is mostly of extraterrestrial in origin $[5,6]$ making it improbable that the BEs we find (which are not associated with terrestrial biology) originate from Earth.
Here we present images of some of the more striking BEs sampled from two separate stratosphere-sampling launches. In all cases the images shown exceed 5 micron in size and most are shown, by EDX analysis, to contain carbon oxygen and no significant amounts of the other elements (e.g. $\mathrm{Ca}, \mathrm{Fe}, \mathrm{Mg}, \mathrm{S}$ and $\mathrm{Si}$ ) which are typically found in inorganic particles derived from space, or Earth.

\section{Materials and Methods}

A balloon-launched sampling device was released and successfully retrieved $[1,2]$ on three occasions. The first launch (June, 2013) was released from near Chester, Cheshire and landed near Wakefield, West Yorkshire. The second launch (August, 2014) travelled from Sheffield and landed near Grimethorpe in West Yorkshire; finally, the third launch was released (January, 2015) from Ashbourne, Derbyshire and landed at Sturston, Derbyshire. In all cases, the sampler was returned to Earth by parachute undamaged and completely intact. The sampler included a drawer mechanism that could be automatically opened and closed at a chosen height (Figure 1). The stratosphere sampler also carried a video camera by which the opening and closing of the sampling drawer could be viewed, confirmed and recorded.

The sampling apparatus was protected from any downfall of particulate matter from the balloon itself by means of a cover. Prior to launch, the inside of the drawer device (Figure 1) was scrupulously cleaned, air blasted and finally swabbed with alcohol. New scanning electron microscope stubs were placed in rows inside the drawer with their top surfaces facing outwards so that when the drawer was opened any particulate matter in the stratosphere would attach to them and they could later be removed for examination under the

*Corresponding author: Wainwright M, Department of Molecular Biology and Biotechnology, University of Sheffield, Sheffield S10 2TN, UK, Tel: 442920752146 ; E-mail: m.wainright@scheffield.ac.uk

Received January 30, 2015; Accepted March 04, 2015; Published March 17, 2015

Citation: Wainwright M, Rose CE, Baker AJ, Wickramasinghe NC, Omairi T (2015) Biological Entities Isolated from Two Stratosphere Launches-Continued Evidence for a Space Origin. Astrobiol Outreach 3: 129. doi:10.4172/2332-2519.1000129

Copyright: ( 2015 Wainwright $\mathrm{M}$, et al. This is an open-access article distributed under the terms of the Creative Commons Attribution License, which permits unrestricted use, distribution, and reproduction in any medium, provided the original author and source are credited. 
Citation: Wainwright M, Rose CE, Baker AJ, Wickramasinghe NC, Omairi T (2015) Biological Entities Isolated from Two Stratosphere LaunchesContinued Evidence for a Space Origin. Astrobiol Outreach 3: 129. doi:10.4172/2332-2519.1000129

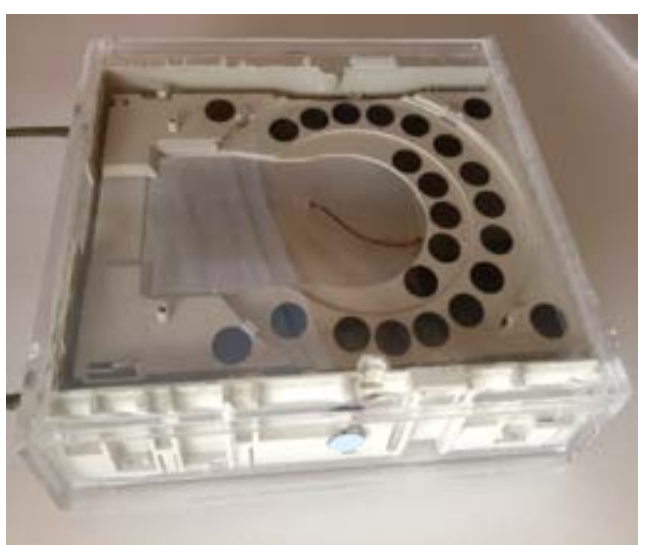

Figure 1: The airtight drawer sampler which was automatically opened and closed in the stratosphere, showing scanning electron microscope stubs onto which stratospheric particles were directly deposited.

scanning electron microscope. The protective layer on the surface of the stub was peeled off just before launching under a cover to prevent any particulate contamination. After sampling, the apparatus was transported to the laboratory and opened under conditions which avoided exposure of the stubs to contaminating dust and the stubs were similarly transferred under cover to the scanning E/M. The stubs were sputter-coated with gold for 30 secs at $30 \mathrm{~mA}$ and then examined using a SEM (JEOL 6500F).

\section{Control flight}

A separate control flight was made to the stratosphere prior to the sampling flight, when the drawer was not opened, but all other sampling procedures were observed. No particulate matter was found (using the SEM) on any of the unexposed microscope stubs in the control, showing that the drawer remained airtight and that none of the stubs was exposed to particles at, or near ground-level or at any height up to the stratosphere. These results also show that no particles contaminated the stubs during any of the sample processing procedures, thereby demonstrating that the scrupulous procedures used to prevent ground-level contamination proved effective, and that no such contamination occurred.

\section{Results and Discussion}

\section{Sampling flight 1 (25-27 Km)}

Figure 1 shows the inside of the sampling drawer and the SEM stubs onto the surface of which, the particles discussed below fell during sampling of the stratosphere. SEM images of contaminating material removed from the outside of the sampler box are shown in Figure 2. Pollen and fungal spores from this source are shown in Figure 2A, while a variety of grass particles is shown in Figure 2B. Such terrestrial materials were never observed on the inside of the sampler or on the sampling stubs and so were not present in the stratosphere. Figure 3 shows craters caused on the sampling stubs by micrometeorite impact events. The presence of such space-derived craters suggests, but does not conclusively prove, that the BEs originated from the same source; micrometeorites (from space) may of course be impacting the stub at the same time as the BEs are up-coming from Earth. Note however, the absence of terrestrial dust or biology near the impact craters (Figure 3 ) on the stub- surface. This begs the question- how could BEs coming up from Earth be associated with micrometeorites, while no obvious biology from the same source is present on the stubs?
A particularly interesting BEs is shown in Figure 4. It possesses a characteristic two-part proboscis-like structure, the bottom of which has an entrance with a nose-like appearance. A structure with the appearance of a sphincter is seen on the top of the biological entity and a flap-like structure on the side. We assume that this BE would, in its normal state, be inflated like a ball with the proboscis pointing downward. Due to bilateral symmetry, we also again assume that the flap-like structure would be replicated on the unseen side of the organism. We speculate that this structure may be used to propel the organism in a liquid environment. Note that here, and elsewhere in
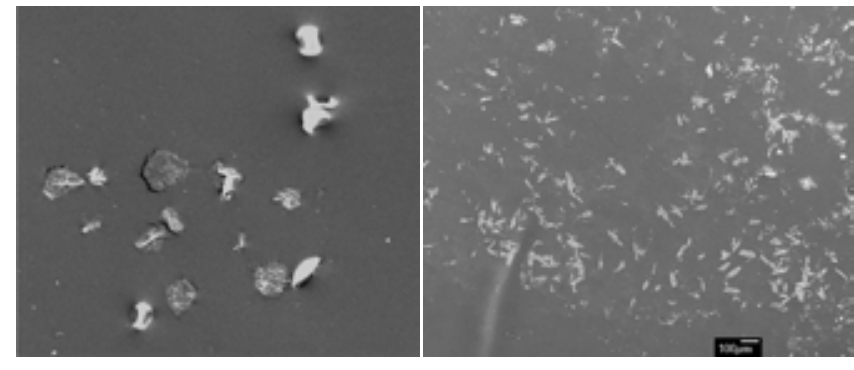

Figure 2A and 2B: Pollen and fungal spores and B, grass particles found only on the outside of the sampler.

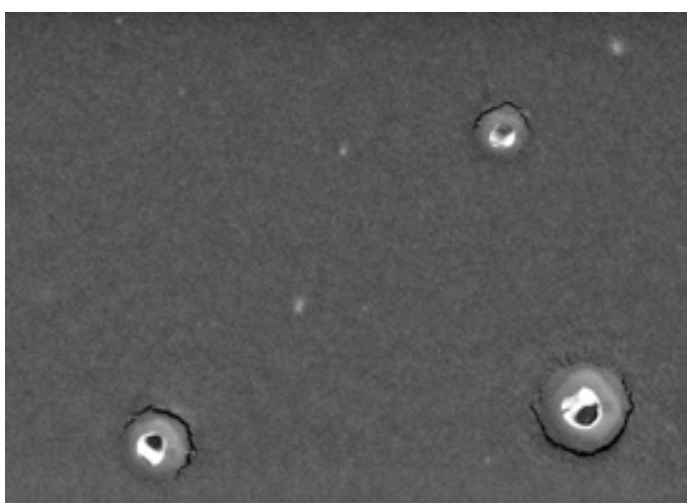

Figure 3: Craters on the sampling stubs resulting from micrometeorite impact.

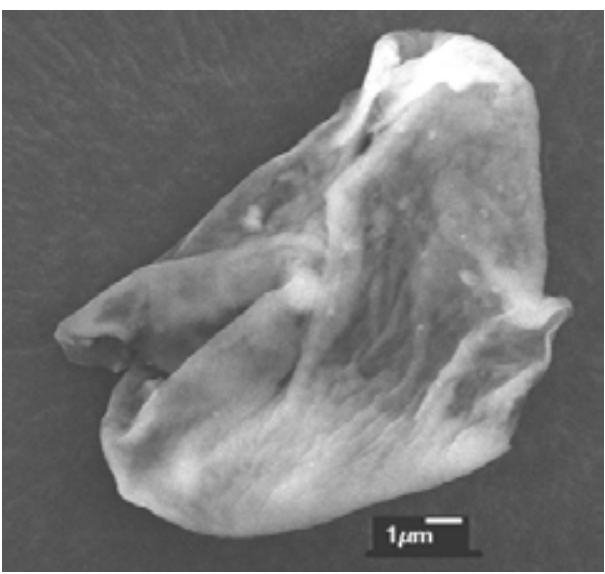

Figure 4: Stratospheric biological entity (BE) showing marked proboscis-like structure (No EDX available). 
Citation: Wainwright M, Rose CE, Baker AJ, Wickramasinghe NC, Omairi T (2015) Biological Entities Isolated from Two Stratosphere LaunchesContinued Evidence for a Space Origin. Astrobiol Outreach 3: 129. doi:10.4172/2332-2519.1000129

this paper, we use familiar terms (such as proboscis-like, or sphincter) to aid our description of the BEs. This does not, of course, necessarily imply correspondence, or similar functionality, between such terms and the morphology-biology of the BEs.

One of the most intriguing BEs (colloquially named by us, the "dragon particle") which we have isolated from the stratosphere is shown in Figure 5. It is not clear whether this complex particle is a single entity, which is part of a larger biological structure, or if it consists of individual organisms attached to a single "back plate". Different observers will see and interpret the component parts of this image in different ways. What is clear is that Figure 5 shows a complex object which is not an inorganic particle mass and which cannot be matched (at least by us) to any known terrestrial, biological structure or mass of individual organisms.

A flask-like structure (which has collapsed during SEM processing) is shown in Figure 6 having a pronounced neck and what appear to be cilia surrounding the neck orifice.

Figure 7 shows a fragment of what is unmistakably a diatom which was isolated at between $25-27 \mathrm{Km}$. This image has resulted in the most opprobrium from our critics who argue that Occam's Razor can be invoked to show that this diatom fragment must have been elevated to this region from Earth, by some unknown mechanism which can lift particles larger than 5 microns to the stratosphere. Our response to this criticism is- if such a mechanism exists, why are there no pollen grains, fungal spores or grass particles (or other readily identified example of Earth-biology) associated with this diatom fragment? This observation, we suggest, allows us to turn Occam's razor on our critics and claim that the diatom fragment arrived into the stratosphere from space.

Figure 8 shows a sphere from which a mucoid material is emerging from an impact event caused when the sphere impacted the carboncoated sampling stub. The sphere was moved away from the impact event using Nano manipulation. The mucoid material is composed of only $\mathrm{C}$ and $\mathrm{O}$ as is the filamentous material found below the surface coating of the sphere. The surface of the sphere is shown by EDX to be titanium with a small amount of vanadium. We conclude that the sphere is rich in titanium and is covered with in biological filaments and contains a mucoid biological material. This image is of fundamental importance because it clearly shows an impact event (i.e. a crater) on the sampling stub, which shows that the sphere must have been incoming to the stratosphere at speed when it collided with the

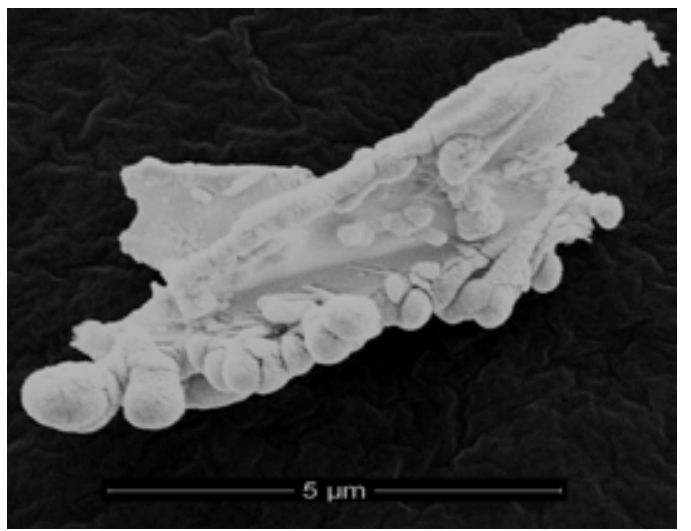

Figure 5: A complex BE isolated from the stratosphere (the so-called "dragon particle") EDX at $\mathrm{C}$ and $\mathrm{O}$ only.

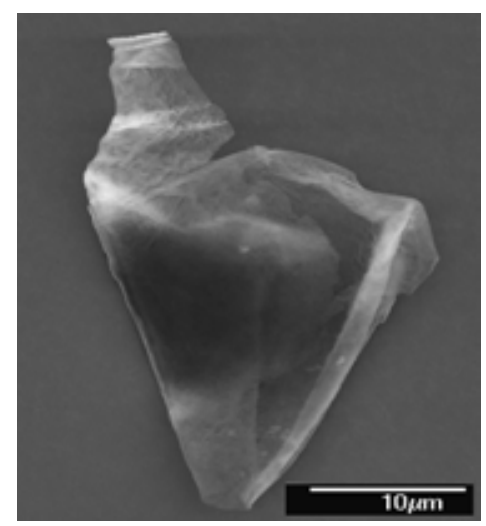

Figure 6: A flask-shaped BE (no EDX available).

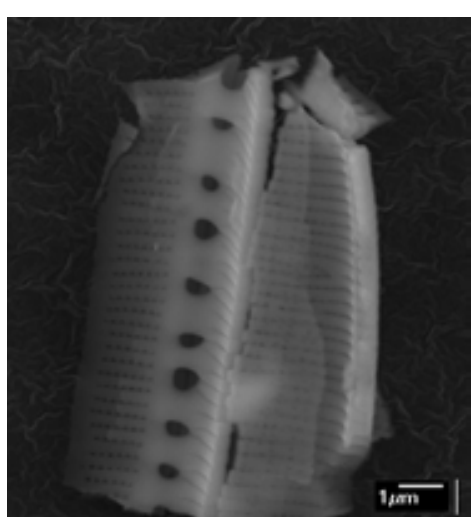

Figure 7: An obvious fragment of diatom frustules sampled from the stratosphere.

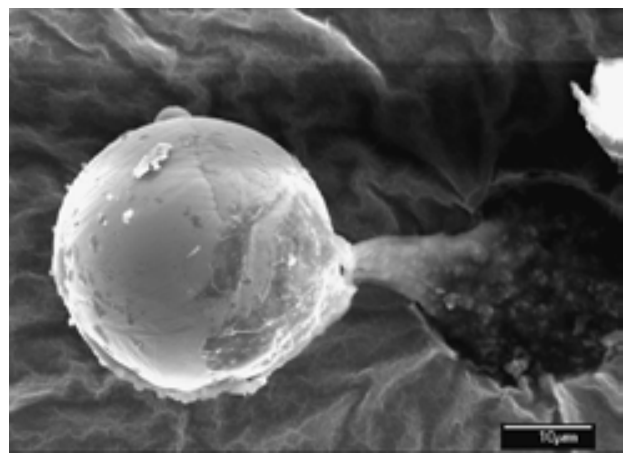

Figure 8: A titanium-vanadium sphere with both biology-like filaments on outside and mucoid material oozing out of sphere EDX at $\mathrm{C}$ and $\mathrm{O}$ only.

sampling stub, i.e. it came from space. If in the unlikely event that the other BEs shown in this paper were shown to be of terrestrial origin Figure 8 would provide almost certain evidence for a space origin for at least one of our BEs.

The biological entity shown in Figure 9 is composed of $\mathrm{C}$ and $\mathrm{O}$ only. It is a complex biological structure with distinct "spiracle-like" pores [1]. This BE is of particular importance because it caused an impact event on the sampling stub, showing that it was travelling at speed when it impacted the sampler (we assume that it was not "naked" 
Citation: Wainwright M, Rose CE, Baker AJ, Wickramasinghe NC, Omairi T (2015) Biological Entities Isolated from Two Stratosphere LaunchesContinued Evidence for a Space Origin. Astrobiol Outreach 3: 129. doi:10.4172/2332-2519.1000129

Page 4 of 5

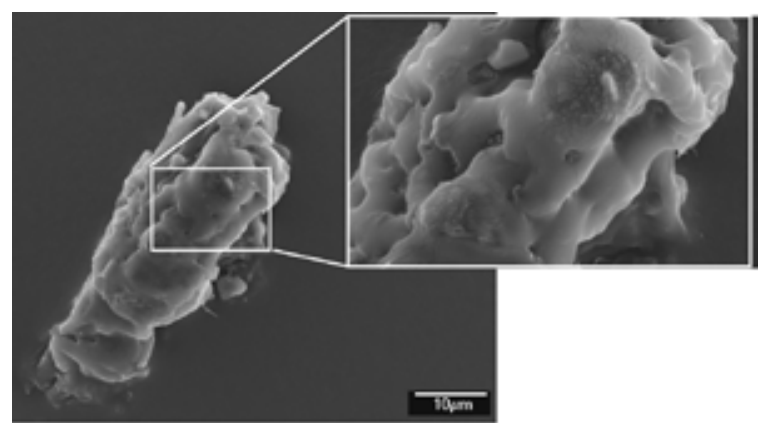

Figure 9: A stratosphere-derived BE (insert shows "spiracle like" openings) (EDX at $\mathrm{C}$ and $\mathrm{O}$ only).

when it impacted. but was enclosed in an ice particle) and so originated from space.

\section{Sampling flight $2(25-27 \mathrm{Km})$}

The second sampling showed the presence of micrometeorite impact events on the sampling stubs, but not BEs. This in itself is significant since, if terrestrial organisms were readily being transferred to the stratosphere from the vast biological pool provided by Earth then common microbes would be found on every sampling trip. The arrival of unusual biology from space, on the other hand, would be expected to occur intermittently (as is the case) depending on the appearance of a delivery system e.g. comets, meteorites or asteroids. It is important to recognize that sampling BEs from the stratosphere is essentially equivalent to a "fishing trip" as a result, confirmation or denial of our claims that unusual BEs exist in the stratosphere cannot be based on a single sampling trip.

\section{Sampling flight $3(23-25 \mathrm{Km})$}

The fact that BEs were isolated on the third successful sampling trip is obviously important since it shows that the sampling of biological entities from the stratosphere on the first sampling trip was repeatable and was not an isolated occurrence.

Figure 10A shows an unusual gossamer-like biological entity isolated from $23-25 \mathrm{Km}$ (which we colloquially refer to as the "ghost particle"). The surface of the gossamer is clearly wrinkled, as if previously expanded. The edge of the gossamer forms a ridge and not a thin edge (Figure 10B) suggesting that this structure is a collapsed balloon-like object, which may previously have been inflated by a liquid or a gas; we speculate that such a "balloon" may have been attached to another part of an organism and acted as a flotation device. We have compared this biological entity, at length, with human skin and dandruff particles, which it could be argued are similar and might have contaminated the sample; we are confident however, that such contamination is not the source of this biological entity.

The bell-shaped biological entity is shown in Figure 11 is difficult to interpret, although it is possibly a piece of the gossamer-like material shown in Figure 10 which has been folded (note, for example, the triangle shape in the foreground of the object) so as to give the artifactual impression of a bell-shaped biological entity.

Finally, Figure 12 shows a star-shaped BE which appears to be made up of sheets of cells fused together, with the sheets decreasing in diameter towards the top-most surface. Some of the cells on the topmost layer are seen to be connected by filament-bridges This BE was found to contain small amounts of sodium; we were however, unable to create a similar structure by allowing various concentrations of sodium bicarbonate and carbonate to form precipitates on drying.

The results of this, third, successful stratospheric sampling trip once again shows that unusual BEs are present in the stratosphere. With the exception of a diatom frustule-fragment found in the first sampling trip, none of these BEs are obviously representatives of known terrestrial biota.

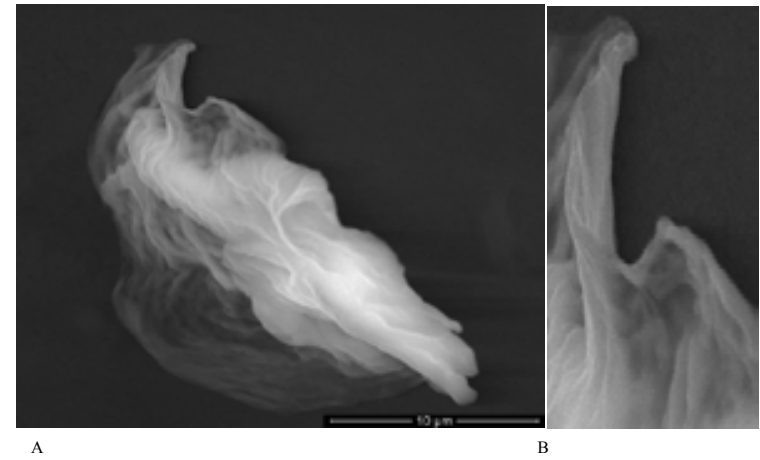

Figure 10: (A) A gossamer-like particle (the so-called "Ghost Particle"), note the thick ridge $(B)$ which suggests that the gossamer is not a paper-thin sheet, but a collapsed balloon-like structure. (EDX at C and O only).

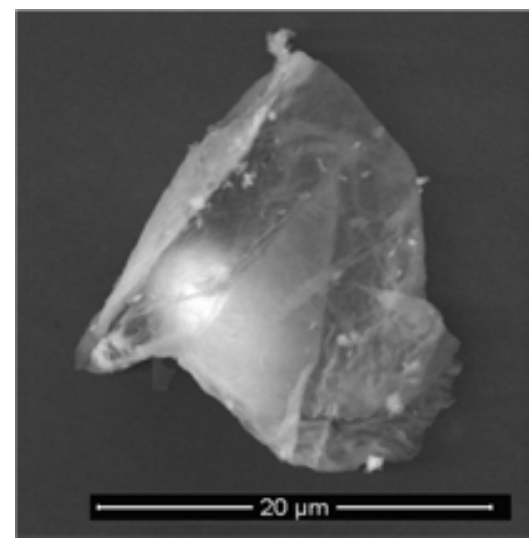

Figure 11: A bell-shaped BE (EDX C and O only).

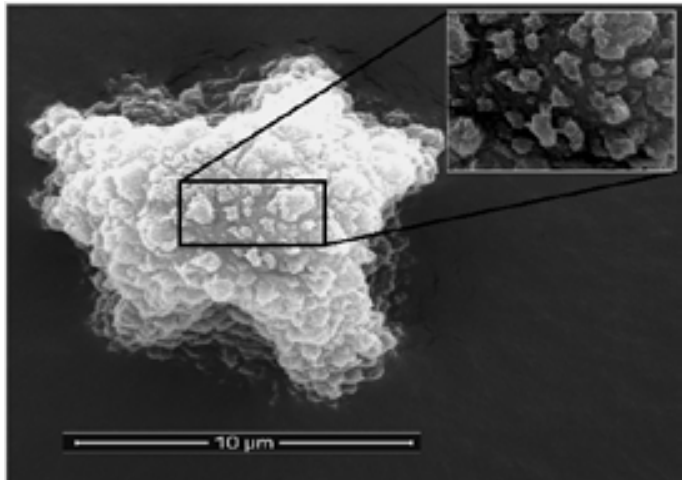

Figure 12: A star-shaped BE with insert showing filament-bridges between particles on the uppermost surface (EDX at $\mathrm{C}$ and $\mathrm{O}$, with trace of $\mathrm{Na}$ ). 
Based on the factors mentioned in the Introduction we remain confident that these biological entities are not being elevated from Earth to the stratosphere, but instead are incoming to Earth from space. One of the principle findings which support this view is the absence of pollen or grass debris on our sampling stubs. In fact the BEs, which are found at very low frequency, occur as individual, pristine, BEs and do not occur with any common Earth-organisms. Pollen and grass debris cover the entirety of the outside of the sampling box (Figure 2) but, as yet, none has been found inside the intact box after its parachute return to Earth. Critics who are convinced that these stratosphere-isolated BEs must (according to the application of Occam's Razor) originate from Earth are asked to describe a mechanism by which such particles are elevated to the stratosphere without any contaminating pollen grains, grass seeds, or marine and fresh water algae or protozoa. What is the nature of the "sieve" that allows such BEs to pass through, while holding back known, obvious terrestrial organisms? We realise that here we are risking becoming "hostages to fortune" since if any stratosphere pollen grains are isolated, either by us or other workers, then this would be taken as evidence that all of our sampled BEs must originate from Earth. However, according to our current understanding, particles greater than 5 micron in size cannot cross through the tropopause into the stratosphere [4]. If a pollen grain or other Earth-derived particle, smaller than this value reached the stratosphere then this would not contradict our suggestion that the BEs originate from space. If however, a pollen grain larger than 5 micron were to be found in the stratosphere then this would demonstrate that an, as yet unknown, mechanism exists which can elevate particles bigger than 5 micron across the tropopause. Such a finding would at first sight destroy our claim that the BEs which we find in the stratosphere originates from space. In this case we would initially fall back on claims of organismnovelty, i.e. that since our BEs (with the exception of the diatom fragment) are morphologically similar to no known biology found on Earth they must originate from elsewhere, an obvious tenuous position since such stratosphere-derived biological entities may exist, undiscovered on Earth (one would still however, ask why it is that common, known organisms, are not over represented on our sampling stubs). The claim that we have discovered biology originating from space would however, continue to be supported by the fact that, in some cases, our stratosphere-isolated biological entities cause impact events on the sampling stubs (see for example, Figure 9), and in the case of a biology-associated titanium ball, a clear impact crater (Figure 8). We assume that such impacts are caused when the BEs reach the surface of the sampling stubs from space inside minute ice grains; it is these we theorize which cause the impact events, only to disappear and leave the pristine BE resting on the impacted carbon surface of the stub. Such impact damage could not be caused by organisms "lazily" drifting up from Earth. They might however, be explained on the basis that an unknown elevation mechanism can carry 5 micron-plus BEs and particle masses, such as the ones we have sampled, from Earth to extreme heights in space.

In conclusion, we remain confident that the evidence continues to show that the increasing number of biological entities which we have isolated from the stratosphere, some of which are described here, originate from space, most likely from comets.

\section{References}

1. Wainwright M, Rose CE, Baker AJ, Bristow KJ, Wickramasinghe NC (2013) Isolation of biological entities from the stratosphere $(22-27 \mathrm{~km})$, Journal of Cosmology 22: 10189-10196.

2. Wainwright M, Rose CE, Baker AJ, Bristow KJ, Wickramasinghe NC (2013) Isolation of a diatom frustule fragment from the lower stratosphere $(22-27 \mathrm{~km})$ Evidence for a cosmic origin, Journal of Cosmology 22: 1083-10188.

3. Taylor HE, Lichte FE (1980) Chemical composition of Mount St. Helens volcanic ash, Geophysical Research Letters 7: 949-952.

4. Rosen JM, Nye EP (1964) The vertical distribution of dust in the stratosphere Smithsonian Contributions to Astrophysics 11: 347-355.

5. Rosen JM (1969) Stratospheric dust and its relationship to the meteoric influx, Space Science Reviews 9: 58-89.

6. Zolensky ME, Mackinnon DR (1985) Accurate atmospheric particle size distributions from a flat plate collection surface. Journal of Geophysical Research 90: 5801-5808. 